\title{
The lipid organisation of the cell membrane
}

\author{
By S. Ladha \\ Institute of Food Research, Norwich Research Park, Colney, Norwich. UK. NR4 7UA. \\ Tel 44(0)1603 255000, Fax 44(0)1603 507723 \\ E-Mail: Ladhas@BBSRC.AC.UK
}

\section{RESUMEN}

\section{Organización lipídica de la membrana celular}

Los lípidos y las proteínas en las membranas biológicas están dispuestos en un mosaico de campos en la membrana. Estos campos representan heterogeneidades a pequeña escala en la composición, forma y fluidez dentro del plano de la membrana, en un rango que va de los cientos de nanómetros a los pocos micrómetros. Estos campos se originan de las complejas interacciones de las mezclas heterogéneas de fosfolípidos, esteroles y proteínas de las que están hechas todas y cada una de las membranas biológicas.

PALABRAS-CLAVE: Campos lipídicos - Composición lipídica - Membrana - Separación de fase.

\section{SUMMARY}

\section{The lipid organisation of the cell membrane}

Lipids and proteins in biological membranes are arranged in a mosaic of domains in the membrane. These domains represent small-scale heterogeneities in composition, shape and fluidity within the plane of the membrane, over the range of hundreds of nanometers to a few micrometers. They arise from the complex interactions of the heterogeneous mixtures of phospholipids, sterols, and proteins that make up all biological membranes.

KEY-WORDS: Lipid composition - Lipid domains Membrane - Phase separation.

\section{INTRODUCTION}

Biological membranes are organised assemblies of lipids, proteins and to a limited extent carbohydrates and are vital to cell function and development. Proteins and polar lipids account for almost all of the mass of biological membranes with a small amount of carbohydatre present as part of glycoproteins or glycolipids.

Each type of membrane (e.g. plasma membrane, mitochondrial, golgi and lysosomal membrane etc) has specialised functions however there are general properties which outline the basic functions of the membrane (Zubay, 1988; Rawn, 1989). These are listed below:

1) Membranes organise biological processes through compartmentalization. Indeed, the cell, a basic unit of life, is essentially defined by its enveloping plasma membrane;

2) The cell uses the membrane to generate a transmembrane gradient for a variety of purposes. For example, the ion gradients involved in instigating the neuronal action potential. Essentially acting as energy transducers by converting one energy form to another;

3) The membrane is used to organise enzymes into a functional complex. For example, the electron transport chain of the inner mitochondrial membrane;

4) The membrane provides an environment for enzymes to function. The function of these enzymes can be regulated by the membrane organisation and by specific interactions of membrane lipids with the enzyme;

5) Membrane lipids act as reservoirs of precursor compounds required for synthesis of complex molecules. For example the synthesis of prostaglandins from arachidonic acid derived from membrane lipids;

6) The membrane is also responsible for the transduction of signals from one side of the membrane to another. The classic example of this is the plasma membrane adenylate cyclase system. A certain class of hormones interact with the receptors on the surface and elicit an intracellular response via the adenylate cyclase system.

7) The membrane is a two-dimensional structure and therefore the efficiency of certain enzyme catalysed reactions will be much higher than in a three dimensional structure due to the increased probability of intermolecular collisions.

The general cellular processes associated with cell membranes that have been described above are underpinned by the physical structure and chemical architecture of the membrane. To fully appreciate membrane function, knowledge of the composition and structure is essential. In this review, the lipid molecular components of the 
mammalian cell membrane and how these are organised, will be described.

\section{CHEMICAL STRUCTURE}

\section{Amphipathic nature of membrane lipids}

The basic structural components of the cell membranes are molecules which are collectively known as lipids (Zubay, 1988). Although lipid molecules display considerable structural diversity (Gurr et al., 1990; Vance et al., 1991) they all share one important property, they are amphipathic. One end of the molecule has a relatively small polar group moiety while the remainder consist of a hydrocarbon moiety that also gives the molecule hydrophobic characteristics. These molecules adopt certain organised structures when in an aqueous environment as a result of the hydrophobic effect (Tanford, 1980). These include the formation of the lipid bilayer.

\section{Glycerophospholipids}

Glycerophospholipids (Gurr et al., 1990) are the most abundant polar lipid in most biological membranes. Their structure consists of two acyl moieties linked via an ester bond to position 1 (sn-1 position) and 2 (sn-2 position) of a glycerol molecule with a polar or charged alcohol group (head group) linked via a phosphodiester bond to position 3 (figure 1).

The acyl moieties attached to position 2 are usually unsaturated. In some cell types (heart tissue and sperm) a high proportion of the saturated fatty acyl moieties are linked via an ether, rather than an ester, bond at position 1 of the glycerol moiety (sn-1 linked) (Horrocks et al., 1982; Paltauf, 1994) (figure 2).
This ether bond can be either an alkylether or an alk-1'-enylether (plasmalogen). The sn-2 position in these ether lipids is still predominately occupied by unsaturated fatty acids attached via the normal ester bond to the glycerol moiety of the phospholipid.

\section{Sphingolipids}

This type of polar lipids are composed of a long-chain fatty acid, usually linked via an amide bond, to the carbon at position 2 of 4-sphingenine (a long-chain amino alcohol sphingosine) or its derivative with a polar alcohol attached to the carbon at position 1 (head group) (figure 3).

The head group is either attached by an ether or a phosphodiester bond and the nature of the head group gives rise to three basic types of sphinogolipids which are sphingomyelin (phospholipid), neutral glycolipids and gangliosides (Gurr et al., 1990).

\section{Sterols}

The basic structure of sterols consist of four fused rings, three with six carbons and one with five carbons (Gurr et al., 1990). These rings form the steroid nucleus which is almost planar. Cholesterol is the major sterol of mammalian plasma membranes and consists of the steroid nucleus, a polar hydroxyl group and a hydrocarbon tail (figure 4).

Although its structure is quite different from the other lipids discussed, it is still amphipathic. Cholesterol plays a vital role in mammalian cells as they cannot grow efficiently without cholesterol in their membranes (Yeagle, 1985).

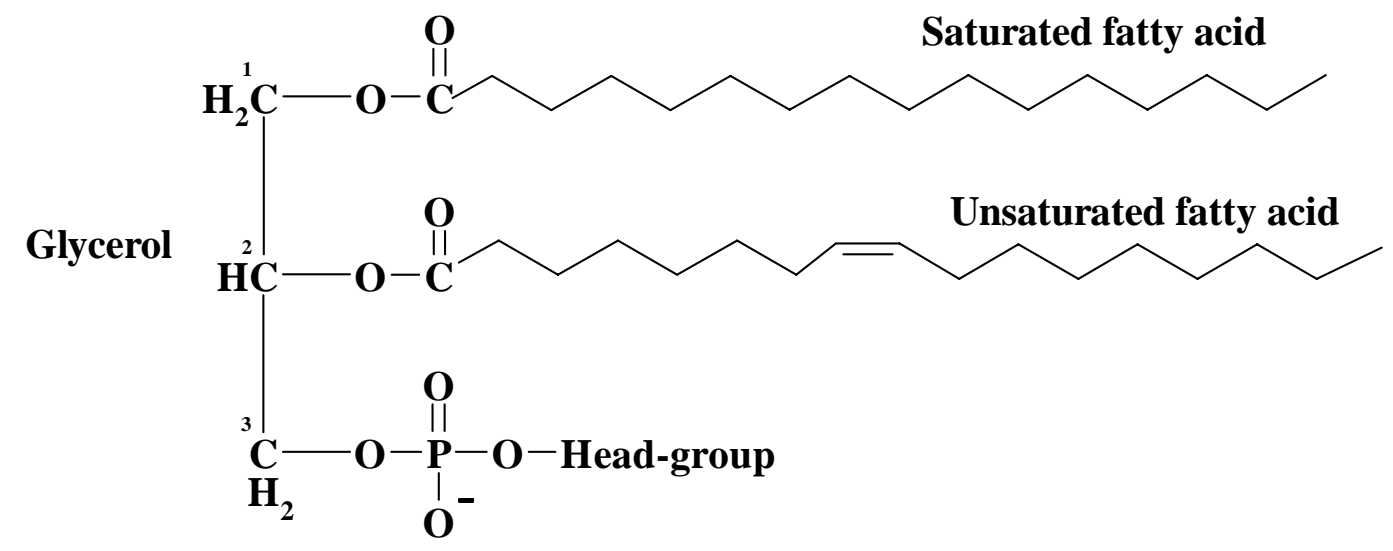




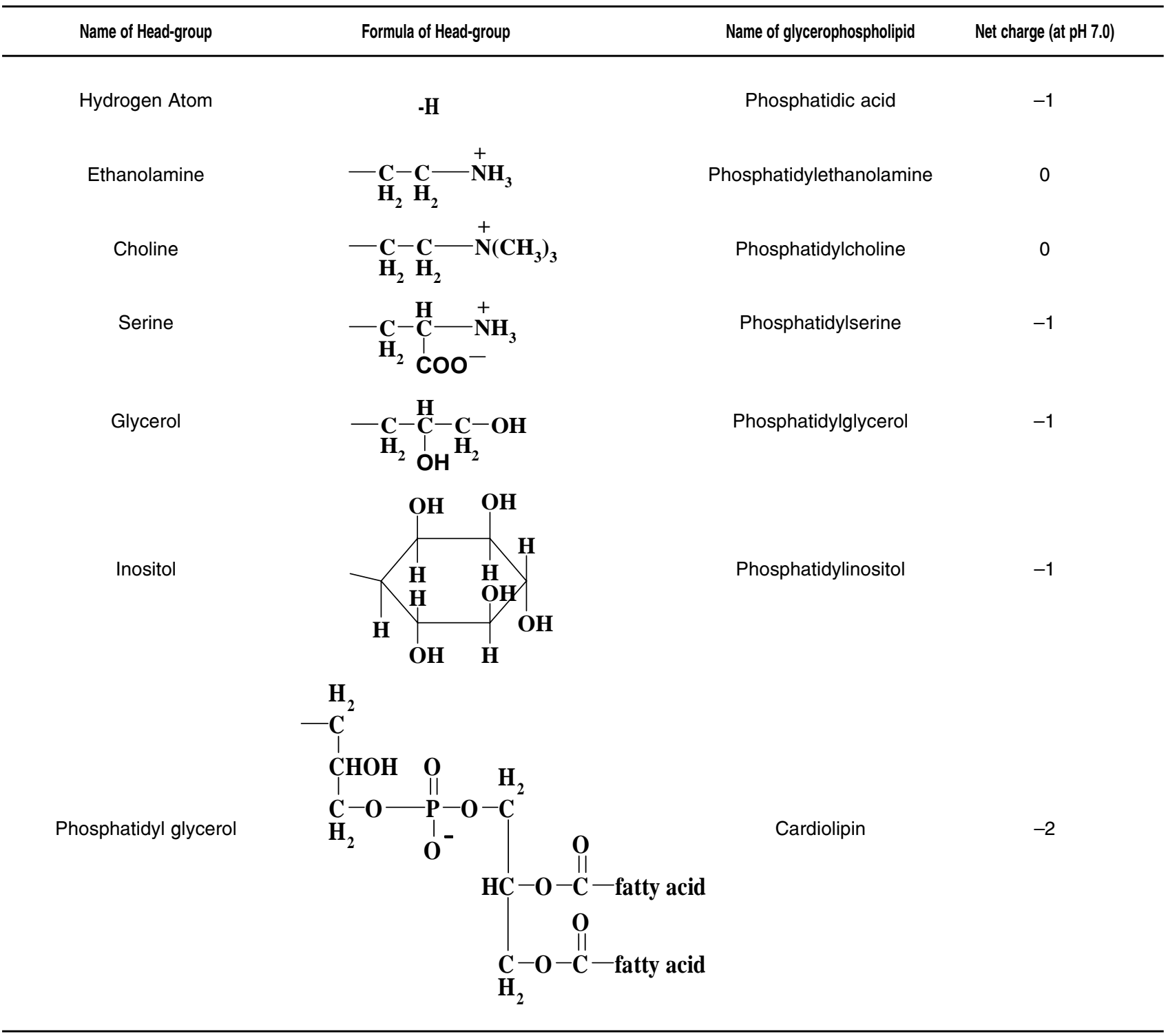

\section{Lipid Composition Complexity}

The above description of lipid composition is by no means comprehensive. However, it is evident that several classes of lipid exist by virtue of the chemical structure of the head group (figure 2 and 3 ). Therefore grouping lipids according to their headgroup structure is one level of complexity. This complexity at the head group level is further exacerbated by the nature of the hydrophobic fatty acyl groups that are attached. These groups can be saturated or unsaturated and generally vary in chain length from 12 to 26 carbons. The unsaturated fatty acyl groups can have up to six double bonds.
This potential variety of possible lipid species leads to the complex lipid composition of cellular membranes. Often membranes from certain sources such as heart tissue and spermatozoa (Ladha, 1998) exhibit a particular composition with respect to lipid classes. The mixture of fatty acids associated with these classes are also characteristic of that class and source of the lipid. The reason why a cell generates such a complex lipid environment in the membrane has not been completely elucidated. It can be argued that this complexity is necessary for membrane function, since the cell would not produce such a complex lipid mixture in the membrane if it was not required. This would require 


\section{1-alkyl-2-acyl glycero-phospholipid}

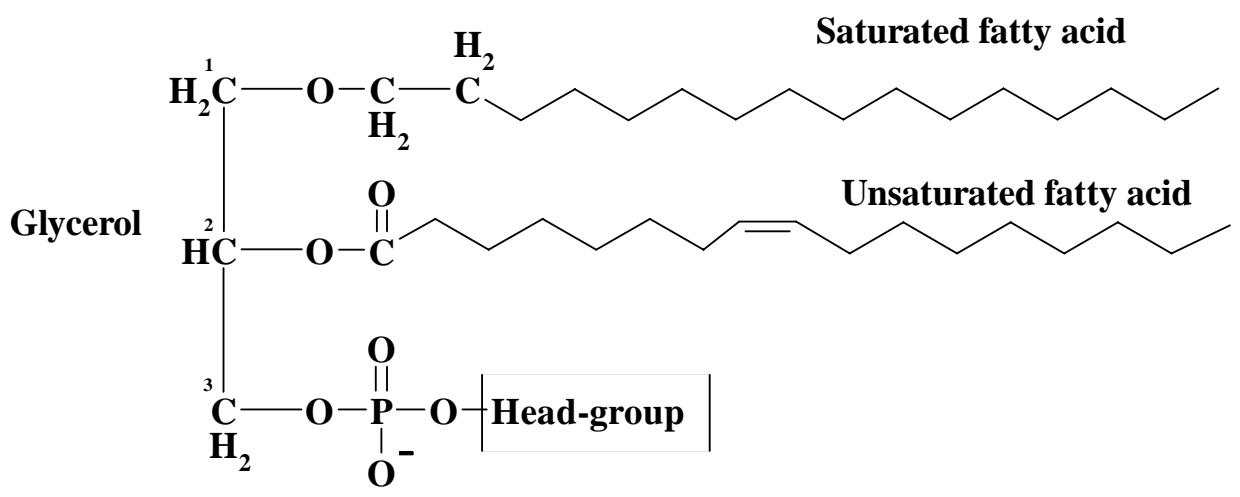

1-alk-1'-enyl-2-acyl glycero-phospholipid( Plasmalogen)

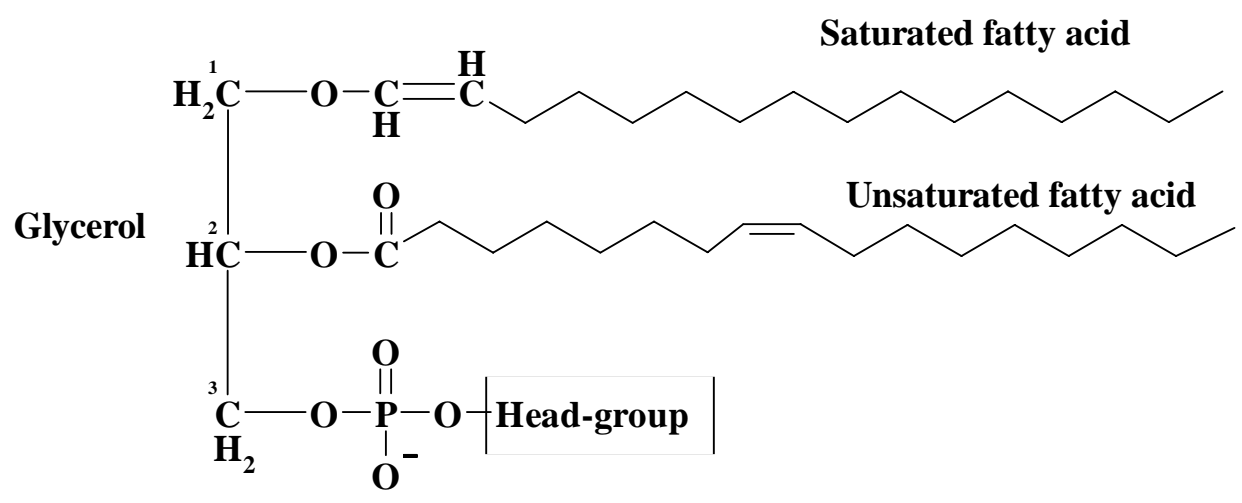

Figure 2

The Ether Linked Glycerophospholipids

the cell to precisely control the membrane lipid composition. However, it can also be argued that rather than the complexity of lipid composition being achieved by the cell, it could be that the cell membrane can function fully with a wide range of lipids, so precise control of lipid composition is not necessary (Lee, 1985). Therefore, a relatively indiscriminate incorporation of fatty acids obtained from the diet, into membrane lipids would be easier than to precisely control the incorporation to produce a membrane containing just a few species of fatty acyl chain (Lee, 1988). Clearly a broad level of control is necessary to maintain the membrane composition, and thereby its physical properties within acceptable limits. For example, the membrane phospholipid fatty acid composition is often re-tailored in response to a change in the external temperature, perhaps in order to maintain the appropriate membrane physico-chemical properties for function (Stubbs and Smith, 1984; Shinitzky, 1984).

\section{MEMBRANE STRUCTURE}

\section{The Bilayer}

A bilayer structure based on amphiphilic phospholipids was first suggested by Gorter and Grendel (1925). This suggestion was based on experiments in which they extracted lipid from human erythrocytes and spread it at an air-water interface. The surface area covered by the lipid was twice that of a single human erythrocytes. From this data they 


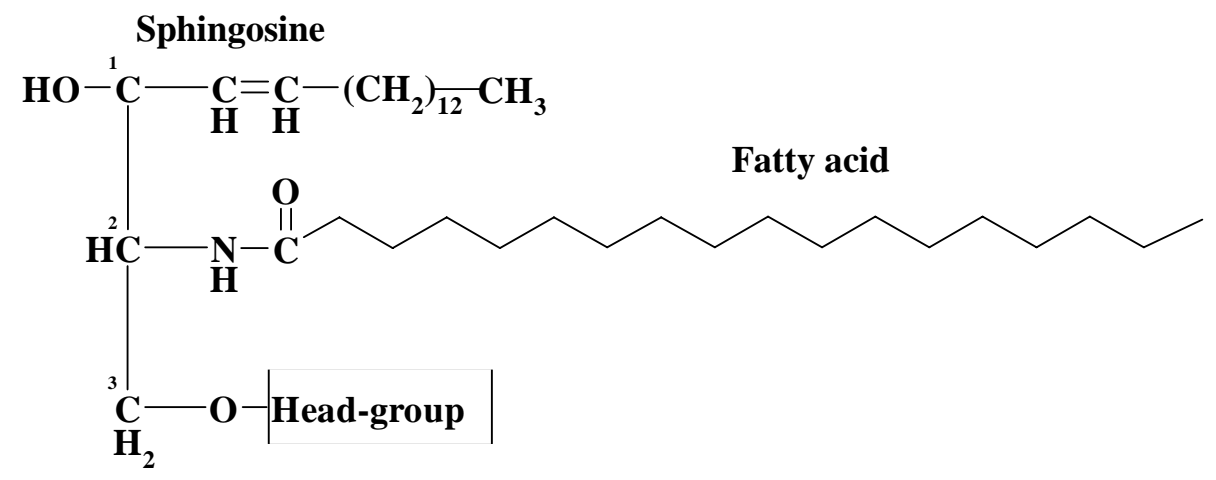

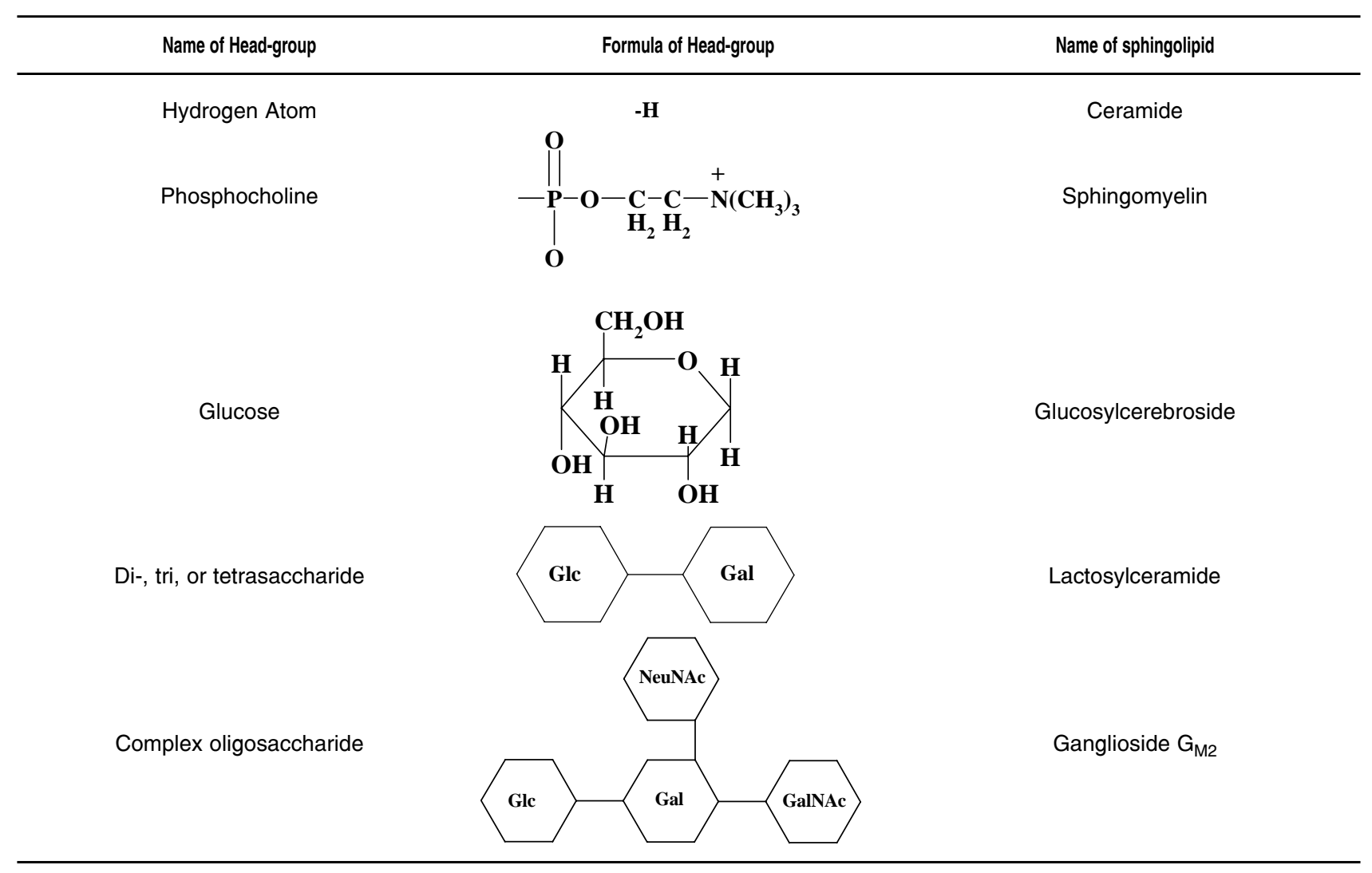

Figure 3

Sphingolipids.

$($ Glc = D-glucose $;$ Gal = D-galactose $;$ GalNAc = N-acetyl-D-galactosamine $;$ NeuNAc = N-acetylneuraminic acid $)$

concluded that a lipid bilayer formed the plasma membrane of the erythrocyte. The subsequent investigators developed this idea and the concept of proteins in the membrane was introduced by Danielli and Davson (1935). However, in the Danielli and Davson model the proteins were suggested to coat the surface of the lipid bilayer to form a trilamellar sandwich. This notion was supported by the earlier idea that the lipid bilayer completely covered the surface of the cell and the only way to accommodate the membrane protein was to have a trilamellar structure. Accumulation of data led to refinements of the model and in 1972 the
Fluid Mosaic model put forward by Singer and Nicolson became widely accepted (Singer et al., 1972). From this model various assumptions could be made:

1) The bilayer behaves like a two dimensional fluid;

2) The proteins can be attached to the surface of the lipid bilayer (peripheral proteins) or are inserted into the bilayer (integral proteins). This bilayer is the permeability barrier of the cell;

3) The physical (motion of fatty acyl groups) and chemical properties ( see above) of the bilayer, can influence the function of the membrane proteins; 


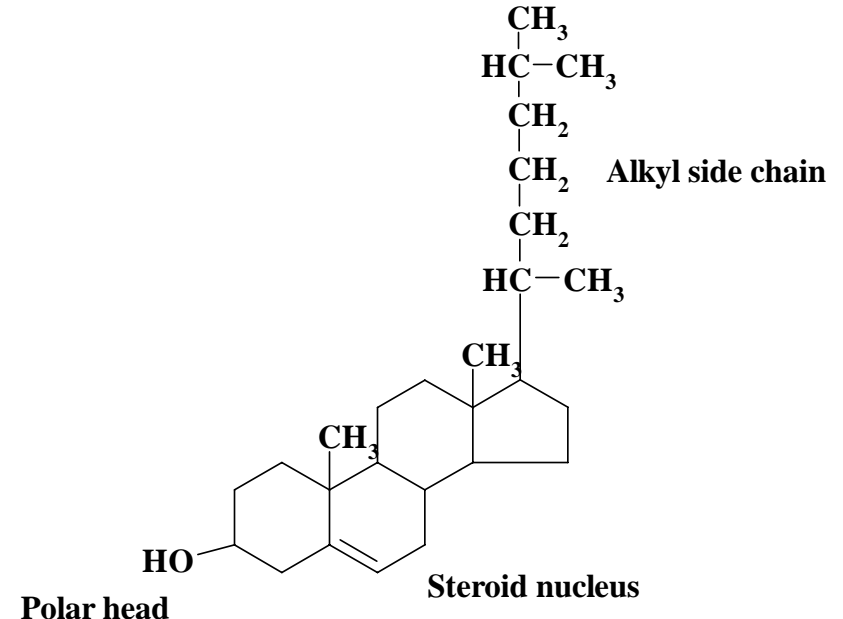

Figure 4 Cholesterol

4) Protein and lipids can diffuse laterally in the plane of the membrane.

\section{Model Membranes}

Liposomes (Bangham, 1968; Ostro, 1987), black lipid membranes (Montal et al., 1972) and lipid monolayers (MacDonald et al., 1987) are three systems that have been extensively used to model membranes. Although they do not exhibit all the complexity of biological membranes, they effectively model some of their properties. These simplified membrane structures are a good approximation to the cell membrane. However, because the composition of the model membrane systems can be controlled, the interpretation of experimental data from a model membrane system is much easier as it is usually composed of only a few known components whereas the native membrane is complex mixture of lipid species and proteins. From studies with model membranes, the interaction of lipid components to influence bilayer behaviour and organisation has been shown to be complex. For example the properties of the lipid component and its behaviour in an aqueous environment can influence the types of lipid assemblies (bilayers phases) that can form. The driving force for lipids to form these assemblies is the hydrophobic effect (Tanford, 1980).

\section{PHASES OF THE MEMBRANE LIPIDS (figure 5)}

\section{Lamellar (bilayer) phase (L $\alpha)$}

Phosphatidylcholine is a lipid commonly found in biological membranes which can adopt a lamellar

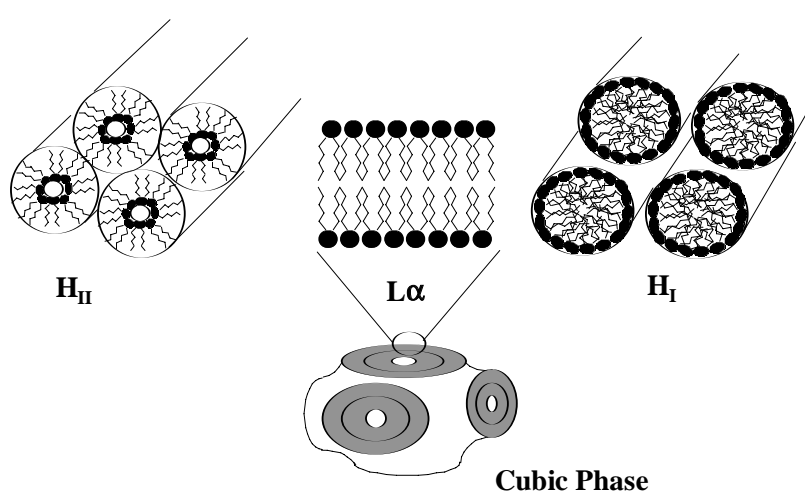

Figure 5

Structures of Four Liquid-Crystalline Phases. ( $L \alpha=$ lamellar (bilayer) phase; $\mathrm{H}_{1}$ and $\mathrm{H}_{\|}=$hexagonal phase)

phase in an aqueous environment (Larsson et al., 1994). This involves the arrangement of the polar head groups such that they face the aqueous environment on both sides of the bilayer and the hydrophobic fatty acyl chains oppose each other inside the bilayer. An interdigitated bilayer is a variation of the lamellar phase that can exist under some conditions. In this situation the ends of the fatty acyl chains from each leaflet of the bilayer are no longer at the midplane of the bilayer but overlap to some degree (Slater et al., 1992).

\section{Non-bilayer phases}

There are two types of hexagonal phases lipids can adopt (Larsson et al., 1994): the hexagonal I phase $\left(H_{1}\right)$; and the hexagonal II phase $\left(H_{\| 1}\right)$. The $H_{1}$ phase exists under specialised conditions and is unlikely to exist in biological membranes. In this phase the lipids form tubes with the polar head groups facing out and the fatty acyl chains facing the interior. Phosphatidylethanolamine is a lipid commonly found in cell membranes which can form the $H_{\|}$phase (Brown et al., 1986). In this phase the lipid forms tubes with the polar head groups facing inside and the fatty acyl chains facing outside (inverse of the $H_{1}$ phase). In cross section a matrix of these structures forms a hexagonal array.

Another phase that can be adopted by lipids is the cubic phase (Lindblom et al., 1989). In this phase the lipids are arranged into short tubes connected in a hexagonal array. These are sometimes found in mixtures of lipids undergoing lamellar to $\mathrm{H}_{\|}$phase transistion (Siegel, 1986).

The significance of having lipids in biological membranes that can form these structures is unclear but it has been suggested that they may be 
involved in the mechanism of membrane fusion (Ellens, et al., 1989; Chernomordik, 1996; Siegel et al., 1997). However, whether these structures exist at all in the complex lipid environment of the membrane is still a matter of debate.

\section{Phase Transitions}

Lipids in model membrane systems (artificial bilayers) undergo phase changes at a temperature (thermotropic phase transition temperature $\mathrm{T}_{\mathrm{m}}$ ) determined by the composition of the bilayer (Melchior et al., 1976). Below the $T_{m}$, the lipids are in the gel phase whereas above, they enter a more fluid liquid crystalline phase. The presence of cholesterol tends to mask the phase changes because it acts as a 'buffer' of fluidity, stiffening the membrane at temperatures above the $T_{m}$ and making it more fluid below the $T_{m}$ (Zubay, 1988).

Another type of transition observed in model membranes is from the $L \alpha$ to the $H\left({ }_{(1}\right)$ phase. Phosphatidylethanolamine (PE) is an example of a lipid found in membranes that undergoes this type of transition (Ellens, et al., 1989). However, significant amounts of the $\mathrm{H}\left({ }_{(I I}\right)$ phase have not been found in biological membranes.

\section{HETEROGENEITY OF BIOLOGICAL MEMBRANES}

\section{Membrane asymmetry}

Although the Fluid Mosaic Model of membranes was a major milestone in membrane research and explained a number of membrane properties, it was however too simplistic. It envisaged that lipids and proteins could freely diffuse within the lateral plane of a simple bilayer structure. The subsequent accumulation of data has demonstrated that the organization of the lipids in membranes is much more complex than that suggested by the original Fluid Mosaic Model of membrane structure. It is now widely recognized that membranes are not homogenous but that lateral asymmetry (Curtain et al., 1988; Tocanne et al., 1989; 1994; Glaser, 1993) and transmembrane asymmetry (Devaux, 1993; Zachowski, 1993) are the rule rather than the exception.

\section{The mosaic of domains (Lateral asymmetry)}

If membrane lipids were allowed to diffuse freely in the plane of the biological membrane then the lipid composition of the surface of the membrane would be homogenous. However, this appears not to be the case. These reasons for this is unclear but this non-uniform distribution is often referred to as lipid domains or microdomains (Thompson, 1993: 1997) in contrast to macrodomains which represent functionally distinct regions on the surface of the cell (eg acrosome, postacrsome, equatorial region, mipiece and principle piece on the mammalian spermatozoa). The commonly used technique to demonstrate lateral heterogeneity in the cell membrane (Ladha, et. al, 1997) is fluorescence recovery after photobleaching (FRAP) (Axelrod et al., 1976). Typically, a small $(1-2 \mu \mathrm{m})$ area of fluorescent-labelled receptors is photobleached by a focused laser beam. The fluorescence in the bleached area is monitored and recovers due to diffusion of unbleached molecules into the area. Recovery is almost always incomplete when measurements are performed on the plasma membrane of living cells. These experiments are conventionally interpreted by two components, a fraction which is immobile on the time scale of the experiment and a mobile fraction which nevertheless is normally characterised by a diffusion coefficient much smaller than expected for unrestricted diffusion. The size and proportion of domains is inferred from the immobile fraction and the bleached area. However, an alternative idea (anomalous diffusion model) has been put forward to account for the immobile fraction detected in FRAP experiments (Feder, et. al., 1996). This suggests that the immobile fraction may actually represent hindered diffusion which on the scale of the FRAP experiment manifests it self as an immobile fraction. Anomalous diffusion in cell membranes may result from diffusing molecules encountering a random array of continuously changing obstacles and traps (binding sites) with a broad distribution of binding energies and escape times (Saxton, 1996).

The mechanism by which microdomains arise has been demonstrated in model membrane systems. For example, in one component lipid bilayers, the gel to liquid crystalline phase transition is sharp i.e. it is either in the gel phase or liquid crystalline phase. However, in a bilayer composed of a mixture of lipids, the phase behaviour can be complex. In essence each species of lipid in the mixture will have different $\mathrm{T}_{\mathrm{m}}$. Therefore, in general, at a low temperature (below $T_{m}$ ) most species of lipid will be in the gel phase while at high temperatures (above $T_{m}$ ) they will be in the liquid crystalline phase. However, at intermediate temperatures some lipid species will be in the gel phase (tempature below $\mathrm{T}_{\mathrm{m}}$ for lipid species) while others will be in the liquid crystalline phase (temperature above $T_{m}$ for lipid species). In this environment gel and liquid crystalline phases coexist and by definition are of different lipid composition. 
In the complex lipid environment of the mammalian membrane it is difficult to prove the coexistence of gel and liquid crystalline domains. However, there is evidences that these type of domains co-exist in gametes (Wolf, 1994) but an alternative suggestion has also been put forward to explain these results (Ladha, et. al., 1997; Ladha, 1998). A more convincing argument for the existence of gel phase lipid domains in cell membranes comes from experiments with a phase sensitive fluorescent probe, lauradan. Using this probe, it was demonstrated that at $37^{\circ} \mathrm{C} 20-32 \%$ of lipids in isolated rat renal epithelial cell brush border membranes were in the gel state (Levi, et. al., 1993). This can be attributed to the high glycolipid and cholesterol content of the apical macrodomain. However, even if the lipids are in the liquid crystalline phase there is a potential for domains to form. This mechanism relies on the immiscibility of the domains and has been demonstrated in model membrane systems containing cholesterol (Sankaram et al., 1991) or glycolipids (Thompson, 1985).

Membrane systems containing a proportion of anionic phospholipids such as phosphatidylserine (PS), are profoundly influenced by calcium. Calcium forms a complex with the negatively charged phosphatidylserine and may induce a phase separation whereby enriched PS domains are formed (Coorssen et al., 1995). These domains may actually enter in to the gel phase due to the partial dehydration of the PS head group by calcium (Roux et al., 1991).

It has also been suggested that possible specialised microdomains composed of cholesterol and sphingomyelin can form within the plane of the membrane (Simons et al., 1997; Harder et al., 1997). This hypothesis put forward recently suggests that the cholesterol and sphingomyelin form rafts in the cell membrane. These may be stablised by a protein called caveolins and together with the rafts of cholesterol and sphingomyelin may be concentrated in flask shaped invaginations called caveolae found in the plasma membrane of some cell types. In this hypothesis one could imagine a membrane consisting of raft and non-raft regions, of which the caveolae are large and stable raft domains. Clearly there will be complex interactive forces which maintain the dynamic equilibrium between raft and non-raft regions. It is purported that these rafts effect membrane function by recruiting specific proteins while excluding others. These rafts therefore serve as platforms to support numerous cellular events in membrane traffic and signal transduction. A large body of evidence for the existence of these rafts comes from experiments which show the that cavelae can be extracted using cold Triton $X 100$. These extracts contain proteins such as glycosylphosphatidylinositol (GPI)-anchored proteins and lipid-linked non-receptor tyrosine kinases. It has been argued that such complexes are artefacts generated by the extraction process. However, direct evidence for the existence of such domains in living cells has been provided recently by Varma and Mayor (1998). In their elegant set of experiments they visualised the domain organisation of the membrane folate receptor (GPI-anchored) using fluorescence resonance energy transfer (FRET) microscopy. The results also showed that cholesterol plays a vital role in organising the domain structure of these proteins.

In addition to lateral asymmetry, the non random distribution of lipids between the two leaflets of the bilayer constitutes a level of transmembrane asymmetry (Devaux, 1993). The two leaflets of the membrane bilayer effectively constitute two macrodomains. Compositional asymmetry between the outer and inner leaflet of the plasma membrane is well established, with clear species-specific patterns. For example, in the erythrocyte membrane it has been shown that PS and $\mathrm{PI}$ and $\mathrm{PE}$ are predominantly located in the inner leaflet where as PC and sphingomyelin are predominantly located in the outer leaflet. Transmembrane asymmetry is established and maintained by an ATP-dependent protein, aminophospholipid translocase (Nolan et al., 1995; Muller et al., 1994, 1996). This enzyme rapidly translocates $\mathrm{PS}$ and $\mathrm{PE}$ from the outer leaflet to the inner leaflet of the membrane (see Zachowski 1993 for general review on mechanism of establishing transmembrane asymmetry). It may be important to the functioning of the leaflets that transmembrane asymmetry provides each side with a different fluidity and microdomain structure.

It must be noted that the model systems used to ascertain the mechanism of domain structure are usually at equilibrium. This means that the extent of phase separation observed may be artifactual compared to the biological membrane which is usually does not operate at equilibrium. It is continuously being perturbed by the incorporation of molecules, protein sorting, lipid sorting and lipid biogenesis. Under these circumstances domain structure will not simply be determined by thermodynamic considerations (Vaz et al., 1993).

\section{CONCLUSION}

Since the advent of the Fluid Mosaic model for the membrane, research into this area has developed these initial ideas further to incorporate the many complexities that were not accounted for by the original model. Recent advances have shown that the complex mixture of lipids and proteins that make-up the membrane are organised into a mosaic of domains. Although there a number 
mechanism by which these can arise, there are still many aspects of membrane organisation which are unclear. For example, the following two questions are germane to this discussion and require further exploration. Firstly, do membrane microdomains form as a consequence of the complex interactions between the many lipid and protein components of the membrane or, is their formation regulated by the cell? The basic thinking behind this question stems from whether the cell simply manufacture membrane lipids with whatever raw materials are available or whether it can sense the microdomain structure of the membrane and make/organise lipids (proteins) that are needed to maintain the microdomain structure. Secondly, what are the function of the membrane microdomains? From the evidence in the literature it is apparent that they provide a specialised (optimal) micro-environment for membrane proteins (Varma et al.,1998) and may therefore help regulate membrane functions.

To further understand the microdomain structure there is a need to harness new methods and techniques (e.g. atomic force microscopy) which will determine directly the microdomain composition and molecular organisation. This would give a better insight into the role of these structures in membrane heterogeniety and function. If the organisation could be probed at the sub-microscopic or molecular level, then the influence of other factors, such as dietary lipid, on microdomain structure could be determined. A recent example of this has been demonstrated by Varma and Mayor (1998) where the microdomain organisation of the folate receptor was shown to be controlled by cholesterol. The consequence of such findings on cellular health remain to be determined but will profoundly affect the understanding of cellular function.

\section{ACKNOWLEDGEMENTS}

The author is supported by funding from the BBSRC.

\section{BIBLIOGRAPHY}

Axelrod, D., Koppel, D.E., Schlessinger, J., Elson, E. and Webb. W.W. (1976). Mobility measurement by analysis of fluorescence photobleaching recovery kinetics. Biophys. J. 16,1055-1069.

Bangham, A. D. (1968). Membrane models with phospholipids. Prog. Biophys. Mol. Biol., 18, 29-95.

Brown, P. M., Steers, J., Hui, S. W., Yeagle, P. L. and Silvius, J. R. (1986). Role of head group structure in the phase behavior of amino phospholipids. 2. Lamellar and nonlamellar phases of unsaturated phosphatidylethanolamine analogues. Biochemistry, 25, 4259-4267.

Chernomordik, L. (1996). Non-bilayer lipids and biological fusion intermediates. Chem. Phys. Lipids, 81, 203-213.
Coorssen, J. R. and Rand, R. P. (1995). Structural effects of neutral lipids on divalent cation-induced interactions of phosphatidylserine-containing bilayers. Biophysical Journal, 68, 1009-1018

Curtain, C. C., Gordon, L.M., and Aloia, R.C. (1988). Lipid domains and relationship to membrane function in Lipid domains in biological membranes: conceptual development and significance, vol. 2, 1-15. Alan r. Liss Inc.

Danielli, J. F. and Davson, H. (1935). A contribution to the theory of permeability of thin films. J. Cell. Comp. Physiol., 5, 495.

Devaux, P. F. (1993). Lipid transmembrane asymmetry and flip-flop in biological-membranes and in lipid bilayers. Current Opinion In Structural Biology, 3, 489-494.

Edidin, M. (1997). Lipid microdomains in cell surface membranes. Current Opinion in structural biology, 7, 528-532

Ellens, H., Siegel, D. P., Alford, D., Yeagle, P. L., Boni, L., Lis, L. J., Quinn, P. J., Bentz, J. (1989). Membranefusion and inverted phases. Biochemistry, 28, 36923703

Feder, T. J., Burst-Mascher, I., Slattery, J. P., Baird, B. and Webb, W. W. (1996). Constrained diffusion or immobile fraction on cell surfaces: A new interpretation. Biophys. J., 70, 2767-2773.

Glaser, M. (1993). Lipid domains in biological-membranes. Current Opinion In Structural Biology, 3, 475-481.

Gorter, E. and Grendel, F. (1925). On bimolecular layers of lipids on the chromocytes of the blood. J. Exp. Med. 41, 439-443

Gurr, M. I. and Harwood, J. L. (1990). Lipid biochemistry. An introduction, 4th Ed., Chapman and Hall, London.

Harder, T, and Simons, K. (1997). Caveolae, DIGs, and the dynamics of sphingolipid-cholesterol microdomains. Current Opinion in structural biology, 9, 534-542

Horrocks, L. A., and Sharma, M. (1982). Plasmalogens and O-alkyl glycerophospholipids in Phospholipids. p. 51-93. J.N. Hawthorne and G. B. Ansell, (Ed.), Elsevier Biomedical Press, Amsterdam.

Ladha, S., James, P.S., Clark, D.C., Howes, E.A., and Jones, R. (1997). Lateral mobility of plasma membrane lipids in bull spermatozoa: heterogeneity between surface domains and rigidification following cell death. J. Cell Science, 110, 1041-1050.

Ladha, S. (1998). Lipid heterogeneity and membrane fluidity in a highly polarized cell, the mammalian spermatozoon. J. Memb. Biol., 165, 1-10

Larsson, K. and Quinn, P. J. (1994). Lipid-water interaction and liquid-crystalline phases in The Lipid Handbook, p. 407-416. F. D. Gunstone, J. L. Harwood, and F. B. Padley (Ed.), Chapman and Hall, London.

Lee, A. G. (1985). Some principles of membrane structure. Proc. Nutr. Soc., 44, 147-156.

Lee, A. G. (1988). Annular lipids and the activity of the calcium-dependent ATPase in Lipid domains in biological membranes: conceptual development and significance. vol. 2, 111-139. Alan r. Liss Inc.,

Levi, M., Wilson, P. V., Cooper, O. J. and Gratton, E. (1993). Lipid phases in renal brush border membranes revealed by laurdan fluorescence. Photochem. Photobiol., 57, 420-425.

Lindblom, G. and Rilfors, L. (1989). Cubic phases and isotropic structures formed by membrane lipids- 
Possible biological relevance. Biochim. Biophys. Acta, 988, 221-256.

MacDonald, R. C., and Simon, S. A. (1987). Lipid monolayers states and their relationships to bilayers. Proc. Natl. Acad. Sci. U.S.A., 84, 4089-4093.

Melchior, D. L. and Steim, J. M. (1976). Thermotropic transitions in biomembranes. Ann. Rev. Biophys. Bioeng., 5, 205

Montal, M. and Mueller, P. (1972). Formation of bimolecular membranes from lipid monolayers and a study of their electrical properties. Proc. Natl. Acad. Sci. U.S.A., 69, 3561-3566.

Muller, K., Pomorski, T., Muller, P., Zachowski, A., and Herrmann, A. (1994). Protein-dependent translocation of aminophospholipids and asymmetric transbilayer distribution of phospholipids in the plasma membrane of ram sperm cells. Biochemistry, 33, 9968-9974.

Muller, K., Pomorski, T., Marienfeld, K., Herrmann, A., and Muller, P. (1996). Transverse mobility of aminophospholipids in the plasma membrane of ram sperm cells. Pflugers Arch., 431, R255-6.

Nolan, J. P., Magargee, S.F., Posner, R.G., and Hammerstedt, R.H. (1995). Flow cytometric analysis of transmembrane phospholipid movement in bull sperm. Biochemistry, 34, 3907-3915.

Ostro, M. J. (1987). Liposomes. Sci. Am., 256, 102-111.

Paltauf, F. (1994). Ether lipids in biomembranes. Chem. Phys. Lipids, 74, 101-139.

Rawn, D.J. (1989). Biochemistry, International Ed., Neil Patterson Publishers, Burlington (North Carolina), p. 209-232.

Roux, M. and Bloom. M. (1991). Calcium-binding by phosphatidylserine headgroups-deuterium NMRstudy. Biophysical Journal, 60, 38-44

Sankaram, M. B. and Thompson, T. E. (1991). Cholesterol-induced fluid phase immiscibility in membranes. Proc. Natl. Acad. Sci. U.S.A., 88, 86868690.

Saxton, M. J. (1996). Anomalous diffusion due to binding: A Monte Carlo study. Biophysical Journal, 70, 12501262

Siegel, D. P. (1986). Inverted micellar intermediates and transitions between lamellar, cubic, and inverted hexagonal lipid phases. Biophys. J., 49, 1155-1170.

Siegel, D. P. and Epand, R. M. (1997). The mechanism of lamellar-to-inverted hexagonal phase transitions in phosphatidylethanolamine: Implications for membrane fusion mechanisms. Biophysical Journal, 73, 3089-3111

Shinitzky, M. (1984). Membrane fluidity and cellular functions. in Physiology of Membrane Fluidity, Vol. I. M.Shinitzky, (Ed.), CRC Press, Inc., Boca Raton, Florida.
Simons, K. and Ikonen, E. (1997). Functional rafts in cell membranes. Nature, 387, 569-572.

Singer, S. J. and Nicolson, G.L. (1972). The fluid mosaic model of the structure of cell membranes. Science, 175, 720-731.

Slater, J. L. and Huang, C. (1992). Lipid bilayer interdigitation in The Structure of Biological Membranes, p. 175-210. P. L. Yeagle (Ed.), CRC Press, Boca Raton.

Stubbs, C. D. and Smith, A.D. (1984). The modification of mammalian membranes polyunsaturated fatty acid composition in relation to membrane fluidity and function. Biochim. Biophys. Acta, 779, 89-137.

Tanford, C. (1980). The hydrophobic effect., Wiley, New York (New York).

Thompson, T. E. (1985). Lipids. Current Opinion In Structural Biology, 3, 473-474.

Thompson, T. E. (1993). Organization of glycosphingolipids in bilayers and plasma membrane of mammalian cells. Annu. Rev. Biophys. Chem., 14, 361-386.

Thompson, T. E. (1997). Lipids: Social order among lipids in membranes. Current Opinion in structural biology, 7, 509-510

Tocanne, J. F., Dupou Cezanne, L., Lopez, A., and Tournier, J.F. (1989). Lipid lateral diffusion and membrane organization. FEBS Lett., 257, 10-16.

Tocanne, J. F., Dupou Cezanne, L., and Lopez, A. (1994). Lateral diffusion of lipids in model and natural membranes. Prog. lipid Res., 33, 203-237.

Vance, D. E. and Vance, J. E. (1991). Biochemistry of lipids, lipoproteins and membarnes in New Comprehensive Biohemistry, Vol 20, Elsevier Science Publishing Co. Inc., New York.

Varma, R. and Mayor. S. (1998). GPI-anchored proteins are organized in submicron domains at the cell surface, 394, 798-801.

Vaz, W. L. C. and Almeida, P.F.F. (1993). Phase topology and percolation in multiphase lipid bilayers - is the biological membrane a domain mosaic. Current Opinion In Structural Biology, 3, 482-488.

Wolf, D. E. (1994). Microheterogeneity in biological membranes. Current Topics in Membranes, 40, 143165

Yeagle, P. L. (1985). Cholesterol and the cell membrane. Biochim. Biophys. Acta Biomembrane Rev. 822, 267 287.

Zachowski, A. (1993). Phospholipids in animal eukaryotic membranes: transverse asymmetry and movement. Biochem. J., 294, 1-14.

Zubay, G. (1988). Biochemistry, 2nd Ed., Macmillan Publishing Company, New York (New York), p. 154-210. 